\title{
Double Nautical Aortic Valve Injury, Secondary to infectious Endocarditis. Case Report
}

\author{
Alicia Santa Cortes González* Britza Barrios Díaz and Héctor Hugo López Medina \\ Instituto Mexicano del seguro social de Xalapa Veracruz, México
}

*Corresponding author: Alicia Santa Cortés González, Servicio de Pediatría HGZ N 11 Instituto Mexicano del seguro social “Dr. Ignacio García Téllez" Lomas del Estadio S/N Col. Centro, Xalapa Veracruz, México

\begin{tabular}{l}
\hline ARTICLE INFO \\
\hline Received: 幽 March 25, 2019 \\
Published: 业 April 04, 2019 \\
\hline Citation: Alicia Santa Cortes González, \\
Britza Barrios Díaz, Héctor Hugo López \\
Medina. Double Nautical Aortic Valve \\
Injury, Secondary to infectious Endocar- \\
ditis. Case Report. Biomed J Sci \& Tech \\
Res 16(5)-2019. BJSTR. MS.ID.002902.
\end{tabular}

\section{ABSTRACT}

Objective: IE is a rare diagnosis in children, although its incidence is increasing, with figures of 0.34 to 0.64 cases per 100,000 inhabitants / year. The NVE has a bimodal distribution with peaks in childhood at 12 to 16 years of age. We present the case of a 14-yearold male with no history of congenital heart disease, respiratory infections, or catheter placement, a course with urinary tract infection treated with cephalosporin, and a month later he developed infectious endocarditis with a double complicated aortic valvular lesion that merited urgent surgical intervention.

Conclusion: Infective endocarditis is rare in children. The presence of persistent fever, heart murmur without structural lesion and antecedent infection should be ruled out.

Keywords: Infective Endocarditis (EI); Native Valve Infection (NVE) Cardiac Vegetation

\section{Introduction}

Infective endocarditis (IE) occurs when any part of the endocardium, particularly the heart valves, is infected by bacteria or fungi [1]. The term NVE is used to describe the infection of a native heart valve in the absence of any prosthetic material. IE is a rare diagnosis in children, although its incidence is increasing, with figures of 0.34 to 0.64 cases per 100,000 inhabitants / year [2]. The NVE has a bimodal distribution with peaks in childhood at 12 to 16 years of age. $90 \%$ of cases of IE occur in individuals with heart disease, usually congenital [3].

\section{Clinical Case}

Male, 14 years old, product of pregnancy 2 without perinatal complications without a history of congenital heart disease, respiratory infections, or catheter placement, with a history of having had urinary tract infection and treatment with cephalosporin for two weeks a month after entering the emergency department reporting asthenia, adynamia, pallor of teguments, chronic fatigue, fever, laboratory studies with microcytic anemia report, hypochromic, hb $9.5 \mathrm{~g} / \mathrm{dl}$, hto $29 \% \mathrm{vcm} 82 \mathrm{fl} \mathrm{HCM} 27.2$ protein c reactive $86 \mathrm{mg}$ / l soft holosistonic murmur grade II DE VI was heard in second right intercostal space, with hyperdynamic precordium, echocardiogram was performed by pediatric cardiology reporting mild pulmonary hypertension, mild mitral regurgitation, with grade 2 diastolic dysfunction, presents pedunculated vegetation in interventricular septum at $55 \mathrm{~mm}$ of valve ao Arctic of $10 \times 12$ bivalve aorta thickened with mild stenosis, pericardial effusion of $170 \mathrm{ml}$, treatment was started for bacterial endocarditis with imipenem / vancomycin for 6 weeks, urine culture was requested and blood culture without development on two occasions, one week later a control echocardiogram was performed, presenting left cavities with a slight dilation of the atrium of $54 \times 54 \mathrm{~mm}$ relation $\mathrm{Ai}$ / Si. 2.5: 1 with dilatation of left ventricle SDVI 11mm LVDD $60 \mathrm{~mm}$ LVDD 43 mm LVEF 53\%.

Atrioventricular valves with $44 \mathrm{~mm}$ mitral annulus with mild mitral regurgitation by color jet and $3 \mathrm{~mm}$ VC, $35 \mathrm{~mm}$ tricuspid annulus with mild tricuspid regurgitation, right ventricular outflow tract without pulmonary artery obstruction, ventricular outflow tract left with double aortic valvular lesion, aortic ring of $21 \mathrm{~mm}$ with GP $8 \mathrm{mmhg}$ and with moderate to severe insufficiency, has two vegetations, one of $12 \times 13 \mathrm{~mm}$ pedicled and located in the interventricular septum and the second one of $25 \times 17 \mathrm{~mm}$ 
in the valve aortic Presents laminar pericardial effusion with distance between sheets of $2 \mathrm{~mm}$, presents secondary arterial hypotension with data of congestive heart failure, is requested assessment by cardiovascular surgery who performs resection of vegetations in aortic valve and aortic valve implant, complicating pericardial effusion requiring pericardial window It presents secondary pericarditis which is managed with non-steroidal antiinflammatory, it complied with the antimicrobial scheme for 6 weeks, with a good evolution, being a hospital graduate with followup by external consultation.

\section{Discussion}

Bacterial endocarditis is an important and complex clinical challenge because the clinical symptoms in children are not specific and can be attributed to other conditions. Nowadays the mortality rate varies between 5 and $10 \%$. Currently, 8 to $10 \%$ of children with infective endocarditis develop without structural heart disease or any other identifiable risk factor. In these situations, the infection usually involves the aortic or mitral valve secondary to bacteremia due to staphylococcus aureus [4]. The presentation in general is indolent, with low and prolonged fever and a variety of somatic complaints, such as fatigue, weakness, arthralgias, myalgias, anemia, weight loss and diaphoresis, that is, the symptoms are very nonspecific. However, sometimes, the presentation may be fulminating, with rapidly changing symptoms and high fevers. These children are seriously ill and some require urgent intervention. In this patient, the initial symptoms were fever with urinary tract infection data, which was treated with antibiotics for two weeks, one month after entering the emergency department with nonspecific symptoms for infection, attention was drawn to anemia without apparent cause and presence of heart murmur, hemoculture and uroculture were performed on two occasions reporting without development being the most common cause of the culture without development the antecedent of therapy with recent antibiotic or an infection caused by an organism such as Abiotrophia and granulicatella species or a HACEK organism that it grows little in vitro [5].

C-reactive protein is elevated in most cases of endocarditis. The transtoracic echocardiogram is the cornerstone to establish the diagnosis has up to $97 \%$ sensitivity for those over 10 years and weighing more than $60 \mathrm{~kg}$ is useful in patients with high risk of abscesses of the aortic root which can complicate the infective endocarditis of the native aortic valve or prosthetic as it happened in this patient, being the first pedicled vegetation and located in the interventricular septum and the second one in the aortic valve being responsible for the double aortic lesion (Figure 1) presenting complications of pericardial effusion, with high risk of vegetation detachment and arterial embolization to any system, showed data of congestive heart failure caused by a worsening of valvular regurgitation accompanied by ventricular dysfunction requiring urgent surgery and resection of vegetations in the aortic valve and aortic valve implant, presenting Plications such as secondary pericarditis and severe peri valvular leakage of $30 \%$ with aortic valve replacement without complications, its initial evolution was insidious, his antimicrobial treatment was completed for 6 weeks with good evolution and he was released to his home.

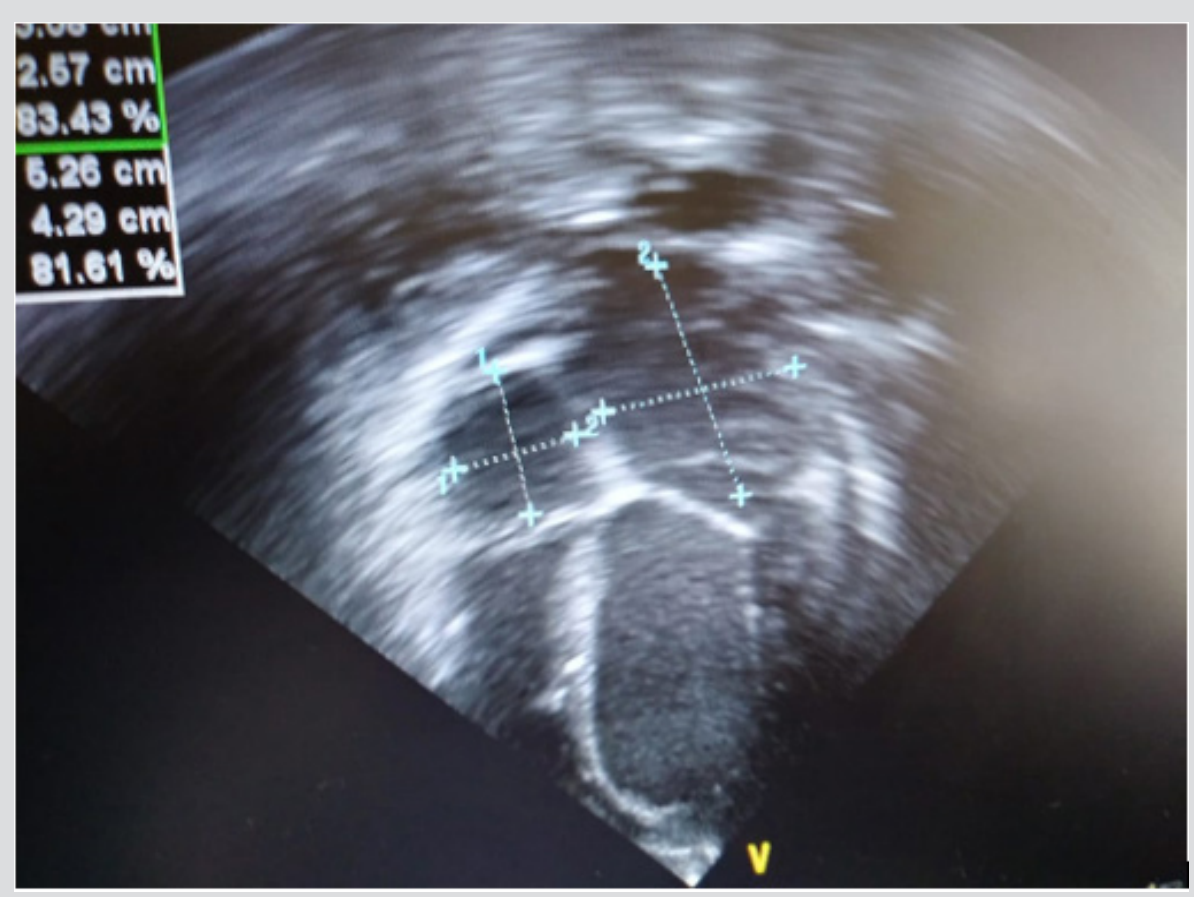

Figure 1: Double lesion aortica. 


\section{Conclusion}

Bacterial endocarditis is an important and complex clinical challenge, because the clinical symptoms in children are not specific and can be attributed to other conditions. The presence of persistent fever, heart murmur without structural lesion and a history of infection makes infectious endocarditis suspect. In this work there are no conflicts of interest or some type of financing. Informed consent was made in this patient.

\section{References}

1. Baltimore R, Gewitz M, Baddour L, Beerman L, Jackson M, et al. (2015) Infective Endocarditis in Childhood: 2015 Update. Circulation 132(15) 1487-1515.

\section{ISSN: 2574-1241}

DOI: 10.26717/BJSTR.2019.16.002902

Alicia Santa Cortes González. Biomed J Sci \& Tech Res

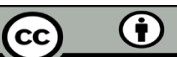

This work is licensed under Creative Commons Attribution 4.0 License

Submission Link: https://biomedres.us/submit-manuscript.php
2. Singh Y, Holme N (2014) Practical guide to managing native valve infective endocarditis in children. Paediatrics and Child Health 24(8): 374-377.

3. Pérez Lescure Picarzo J, Crespo Marcos D, Centeno Malfaz F (2014) Guía clínica para la prevención de la endocarditis infecciosa. Anales de Pediatría 80(3): 187.e1-187.e5.

4. Kelchtermans J, Grossar L, Eyskens B, Cools B, Roggen M, et al. (2018) Clinical Characteristics of Infective Endocarditis in Children. The Pediatric Infectious Disease Journal, p. 1-24.

5. Qureshi N, Patel E (2018) Aerococcus urinae as the Causative Agent in Infective Endocarditis of the Aortic Valve in a Pediatric Patient. The Pediatric Infectious Disease Journal 37(10): 1065-1066.

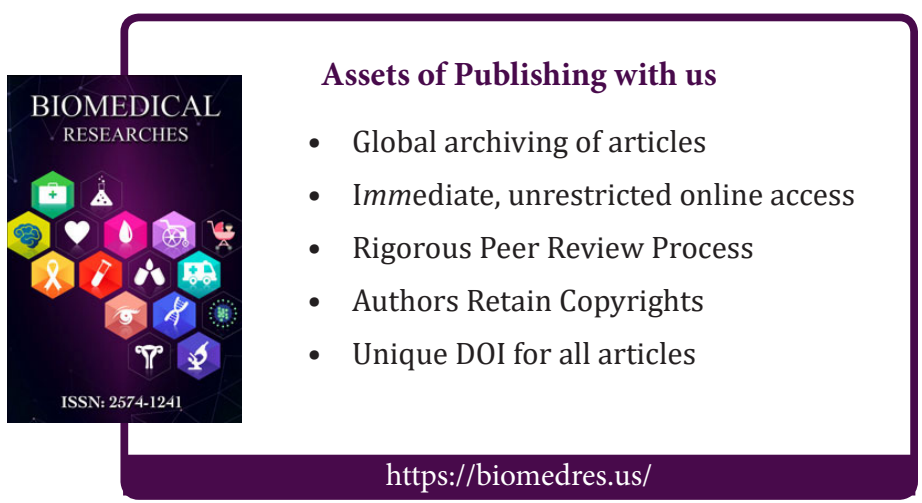

